\title{
Cardiothoracic
}

Transplantation

\section{Life-supporting function of genetically modified swine lungs in baboons}

Bao-Ngoc H. Nguyen, MD, ${ }^{a}$ Agnes M. Azimzadeh, PhD, ${ }^{a}$ Tianshu Zhang, MD, PhD, ${ }^{a}$ Guosheng Wu, MD, PhD, ${ }^{a}$ Henk-Jan Shuurman, PhD, ${ }^{\mathrm{b}}$ David H. Sachs, MD, ${ }^{\mathrm{c}}$ David Ayares, $\mathrm{PhD},{ }^{\mathrm{d}}$ James S. Allan, MD, ${ }^{\mathrm{c}}$ and

Richard N. Pierson III, MDa

From the University of Maryland and Baltimore Veterans Administration Medical Center, Baltimore, $\mathrm{Md}^{\mathrm{a}}$; Immerge Biotherapeutics Inc, Cambridge, Mass ${ }^{\mathrm{b}}$; Transplantation Biology Research Center, Massachusetts General Hospital, Boston, Mass ${ }^{\mathrm{c}}$; and Revivicor, Blacksburg, Va. ${ }^{\mathrm{d}}$

This work was supported by National Institutes of Health U01 AI 066335 (Dr Pierson), NIH R01 HL67110 (Dr Allan), and a Veterans Administration Merit Award to Dr Pierson; F32 HL079818 and Thoracic Surgery Foundation for Research and Education resident research awards to $\mathrm{Dr}$ Nguyen and Dr Stefan Pfeiffer; Deutsche Forschungsgemeinschaft research award to Dr Schroeder; and an Other Tobacco Related Diseases research grant from the Maryland Cigarette Restitution Fund Program.

David Ayares reports equity options in Revivicor, the producer of knockout swine.

Read at the Eighty-sixth Annual Meeting of The American Association for Thoracic Surgery, Philadelphia, Pa, April 29-May 3, 2006.

Received for publication April 28, 2006; revisions received Nov 10, 2006; accepted for publication Nov 20, 2006.

Address for reprints: Richard N. Pierson III, MD, Division of Cardiac Surgery, University of Maryland, $22 \mathrm{~S}$ Greene St N4W94, Baltimore, MD 21201 (E-mail: rpierson@smail.umaryland.edu).

J Thorac Cardiovasc Surg 2007;133:1354-63 $0022-5223 / \$ 32.00$

Copyright (C) 2007 by The American Association for Thoracic Surgery

doi:10.1016/j.jtcvs.2006.11.043
Objective: During ex vivo perfusion with human blood, homozygous galactosyl transferase knockout swine lungs exhibit prolonged survival ( $\sim 2$ hours) relative to wild-type $(<15$ minutes) and swine lungs expressing human decay accelerating factor ( $<1$ hour). In this study, the in vivo behavior of galactosyl transferase knockout lungs was evaluated.

Methods: Three galactosyl transferase knockout swine left lungs were transplanted into baboons in a life-supporting model. One baboon lung allograft and two swine lung xenografts transgenic for human membrane cofactor protein (CD46) served as controls.

Results: Whereas two membrane cofactor protein lungs exhibited high pulmonary vascular resistance $(>500 \mathrm{~mm} \mathrm{Hg} \cdot \mathrm{min} / \mathrm{L})$ and failed to support life within 21 minutes, two of three galactosyl transferase knockout lungs supported life, for 90 and 215 minutes, and displayed low peripheral vascular resistance $(48 \pm 12 \mathrm{~mm} \mathrm{Hg}$ - $\min / \mathrm{L}$ at 60 minutes), similar to the allogeneic control. Complement activation (delta $\mathrm{C} 3 \mathrm{a}<250 \mathrm{ng} / \mathrm{mL}$ through 60 minutes) and C5b-9 deposition were minimal in both galactosyl transferase knockout and membrane cofactor protein lungs. Neutrophils, monocytes, and platelets were rapidly sequestered in galactosyl transferase knockout and human membrane cofactor protein lung recipients, unlike the allogeneic control $(<20 \%)$; and thrombin formation (delta plasma fraction $1+2>$ $0.5 \mathrm{nmol} / \mathrm{L}$ ) was seen in the galactosyl transferase knockout recipients. Platelet activation $(\beta$-thromboglobulin rise $>200)$ and appearance of capillary congestion and vessel thrombosis confirmed coagulation activation associated with galactosyl transferase knockout lung failure.

Conclusions: Galactosyl transferase knockout swine lungs are significantly protected in vivo from the physiologic consequences (increased pulmonary vascular resistance, capillary leak) associated with hyperacute lung rejection. As during ex vivo perfusion, dysregulated coagulation - thrombin elaboration, platelet activation, and intravascular thrombosis-mediates galactosyl transferase knockout lung xenograft injury.

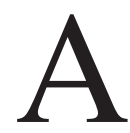
dvances in surgical technology, critical care, and immunosuppression have markedly improved both short- and long-term survival of solid-organ transplants. However, the shortage of human organ donors remains a major limitation in the field of transplantation. Considerable progress has been made in the 


$$
\begin{aligned}
& \text { Abbreviations and Acronyms } \\
& \begin{aligned}
\beta \mathrm{TG} & =\text { beta-thromboglobulin } \\
\mathrm{ELISA} & =\text { enzyme-linked immunosorbent assay } \\
\mathrm{F} 1+2 & =\text { fragments } 1+2 \\
\mathrm{GalT}-\mathrm{KO} & =\text { galactosyl transferase knockout } \\
\mathrm{hDAF} & =\text { human decay accelerating factor } \\
\mathrm{hMCP} & =\text { human membrane cofactor protein } \\
\mathrm{MCP} & =\text { membrane cofactor protein } \\
\mathrm{PVR} & =\text { peripheral vascular resistance }
\end{aligned}
\end{aligned}
$$

Although our ex vivo lung perfusion model is widely established and simulates most physiologic in vivo conditions, exposure of blood to artificial surfaces and concomitant intensive anticoagulation introduce potentially confounding variables. Thus, this in vivo study, the first to describe GalT-KO swine lung function in nonhuman primates, was designed to determine whether the observations ex vivo predict in vivo performance, and specifically to determine whether a GalT-KO lung xenograft is able to support the life of a nonhuman primate.

\section{Materials and Methods Animals}

Genetically engineered swine expressing human membrane cofactor protein (hMCP; $\mathrm{n}=2,60 \mathrm{~kg}$ ) were provided by the Mayo Clinic (Rochester, Minn). GalT-KO swine (20-60 kg) were supplied by Immerge, Inc ( $\mathrm{n}=2$; Boston, Mass) and Revivicor Inc ( $\mathrm{n}$ = 1; Blacksburg, Va). Six Papio anubis baboons (21-25 kg; Buckshire Corporation, San Antonio, Tex) served as lung recipients; and a baboon that had remotely rejected swine heart xenograft was a lung allograft donor. All procedures were approved by the University of Maryland Animal Care and Use Committee and were in compliance with guidelines from NIH publication 86-23, the "Guide for the Care and Use of Laboratory Animals."

\section{Lung Transplant Model}

GalT-KO lungs were harvested remotely either in Boston or Pittsburgh and transported with a cold ischemic time of approximately 6 hours. Two hMCP swine lung donors and one baboon lung donor were sedated with $20 \mathrm{mg} / \mathrm{kg}$ ketamine and $1 \mathrm{mg} / \mathrm{kg}$ xylazine (Phoenix Pharmaceutical Inc, St Joseph, Mo) and given diphenhydramine (Benadryl; Pfizer, Morris Plains, NJ) 10 to $25 \mathrm{mg}$ and ranitidine $2 \mathrm{mg} / \mathrm{kg}$. Anesthesia was induced and maintained with isoflurane $1 \%$ to $3 \%$. After median sternotomy and vascular isolation, heparin was administered (500 IU/kg), and an aortic cannula was secured in the pulmonary artery. Next, 1-benzylimidazole $(5 \mathrm{mg} / \mathrm{kg}$, a thromboxane synthase inhibitor; Sigma No. 116416, St Louis, Mo) and prostacyclin analog (Flolan $0.03 \mathrm{mg} / \mathrm{kg}$; GlaxoSmithKline, Research Triangle Park, NC) were injected by slow bolus infusion into the right heart. Cardiac inflows were occluded, and the lungs were flushed with cold $\left(4^{\circ} \mathrm{C}\right)$ Perfadex (Vitrolife, Kungsbacka, Sweden) containing Flolan $0.5 \mathrm{mg} / \mathrm{L}$ and nitroglycerin $10 \mathrm{mg} / \mathrm{L}$. Cold saline slush was applied to the lungs. After excision of the heart-lung block, the left lung was prepared for transplant into the baboon, and the right lung was prepared for ex vivo perfusion with heparinized fresh human blood as previously described. ${ }^{19}$

Baboon recipients were sedated with $10 \mathrm{mg} / \mathrm{kg}$ ketamine, intubated, and maintained under general anesthesia with isofluorane $1 \%$ to $3 \%$. A femoral arterial line, a femoral vein sheath, and additional intravenous access were secured. Heparin was bolused at $70 \mathrm{IU} / \mathrm{kg}$ and then infused at $200 \mathrm{U} / \mathrm{h}$ to maintain activated clotting time greater than 200. The recipients were treated with diphenhydramine $(0.50-1 \mathrm{mg} / \mathrm{kg})$, ranitidine $(2 \mathrm{mg} / \mathrm{kg})$, and 1benzylimidazole $(7 \mathrm{mg} / \mathrm{kg}$ ). Nitric oxide (INO Therapeutics, Clinton, $\mathrm{NJ}$ ) was delivered to the inspiratory limb of the ventilator circuit and calibrated to deliver $40 \mathrm{ppm}$. One recipient of an hMCP and hDAF lungs were observed, lung fail nevertheless occurred after an average of 2 hours resulting from loss of pulmonary vascular barrier function (pulmonary edema) and/or intravascular thrombosis. 
lung was additionally treated with bivalirudin (Hirulog; The Medicines Company, Cambridge, Mass) $1 \mathrm{mg} / \mathrm{kg}$ bolus before graft revascularization and continuous infusion of $2.5 \mathrm{mg} \cdot \mathrm{kg}^{-1} \cdot \mathrm{h}^{-1}$. A clamshell incision was made at the fourth intercostal space. A flow-directed catheter introduced from the femoral vein was positioned in the pulmonary artery with assistance of direct cardiac manipulation. The right pulmonary artery was isolated with a snared vessel loop, the left hilar structures dissected and occluded, and the left lung removed. The bronchial anastomosis was performed first, followed by arterial and venous anastomoses. Just before revascularization, methylprednisolone (SoluMedrol, $10 \mathrm{mg} /$ $\mathrm{kg}$; Pfizer-Pharmacia, Morris Plains, NJ), C1 esterase inhibitor (Behrinert, 50IU/kg; Behringer), and 1- benzylimidazole $(2.5 \mathrm{mg} /$ $\mathrm{kg}$; Sigma) were given to the recipients by slow bolus infusion. Before and after graft reperfusion, blood and tissue biopsy specimens were collected serially at predetermined time points for in vitro analysis. Vascular flow probes (Transonic Systems Inc, Ithaca, NY) on the aorta and left pulmonary artery continuously measured cardiac output and flow to the transplanted lung, respectively. Thirty minutes after graft reperfusion, flow to the right (native) lung was progressively occluded by snaring the right pulmonary artery to assess the capacity of the transplanted lung to support life. If the animal was unstable with right pulmonary artery occlusion, the right pulmonary artery snare was released, and hemodynamic support was initiated with titrated norepinephrine, vasopressin, and dopamine. The right pulmonary artery was resnared at $15-$ to 30-minute intervals for 90 to 120 minutes, and hemodynamics was recorded; if improvement in lung function was not observed on serial assessment, the experiment was terminated. Graft survival was defined as duration of life-supporting lung function.

\section{Histology and Immunochemistry}

Serial lung biopsy specimens after reperfusion and multiple terminal samples were obtained, trisected, and processed as follows. Formalin-fixed, paraffin-embedded sections were stained with hematoxylin and eosin for light microscopy and analyzed qualitatively for intravascular thrombosis and interstitial, alveolar, or larger airway edema and hemorrhage. Two other pieces were snap-frozen in liquid nitrogen, one without further manipulation and the other after gentle syringe instillation into a visible bronchiole of OCT diluted with saline using a small-gauge angiocatheter. Frozen tissue sections from OCT-infused biopsy specimens were assessed by immunohistochemistry as previously described ${ }^{4}$ with monoclonal antibodies against human C5b-9 (Dako, Copenhagen, Denmark) at 1:50, C4d at 1:50 (Quidel Corporation, San Diego, Calif), CD41 (Immunotech, Marseilles, France) at 1:100, Cy3-labeled goat-anti-mouse immunoglobulin G (Jackson Laboratories, West Grove, Pa), rabbit anti-von Willebrand factor antibody (Dako), and Cy2-labeled goat-anti-rabbit immunoglobulin G antibody (Jackson Laboratories). The deposition of platelets in lung tissue was judged by the staining intensity (from - to +++ ), taking into account the extent and intensity of staining from a standard control section.

\section{Beta-thromboglobulin, Thrombin, and Complement Enzyme-linked Immunosorbent Assays}

Beta-thromboglobulin $(\beta \mathrm{TG})$ and prothrombin fragments $1+2$ $(\mathrm{F} 1+2)$ were measured by commercial enzyme-linked immu- nosorbent assay (ELISA; Asserachrome- $\beta$ TG; Diagnostica Stago, Parsippany, NJ; and Enzygnost micro F1+2; Dade Behring, Marburg, Germany) in plasma samples collected in CTAD tubes (Becton Dickinson, Franklin Lakes, NJ; BD No. 367947) containing $0.109 \mathrm{~mol} / \mathrm{L}$ buffered sodium citrate, $15 \mathrm{mmol} / \mathrm{L}$ theophylline, $3.7 \mathrm{mmol} / \mathrm{L}$ adenosine, and $0.198 \mathrm{mmol} / \mathrm{L}$ dipyridamole. C3a was measured by ELISA (C3a ELISA; Quidel, San Diego, Calif) in plasma samples stored in ethylenediaminetetraacetic acid at $-70^{\circ} \mathrm{C}$.

\section{Flow Cytometry Staining of Platelet Activation}

Blood samples collected in CTAD tubes were immediately fixed with HEPES-buffered saline plus $1 \%$ paraformaldehyde to prevent in vitro activation. Platelet activation was detected by detecting the expression of CD62P by flow cytometry as previously described. ${ }^{5}$ Owing to technical difficulties with sample processing and platelet staining protocols, these data are available for only the last two GalT-KO lung xenograft experiments and for the allograft study.

\section{Hematologic Analysis}

White blood cells, neutrophils, monocytes, and platelets were enumerated by both standard automated (Cell-Dyn; Abbott, Abbott Park, Ill) and manual differential techniques in blood samples collected in ethylenediaminetetraacetic acid.

\section{Results \\ Lung Survival and Function}

The baboon recipient of an allogenic lung exhibited normal physiologic flow to the transplanted lung on revascularization $(0.8 \mathrm{~L} / \mathrm{min})$ and was able to support life with the right pulmonary artery occluded for more than 24 hours, until elective termination of the experiment. As shown in Figure 1, $A$, and summarized in Table 1, PVR was stable throughout the experiment around $30 \mathrm{~mm}$ $\mathrm{Hg} / \mathrm{L} / \mathrm{min}$. Arterial oxygen tension measured from arterial blood ranged from 400 to $600 \mathrm{~mm} \mathrm{Hg}$ on $100 \%$ oxygen.

The recipient of hMCP transgenic swine lung without thrombin inhibition had minimal transpulmonary flow $(<10 \%$ of cardiac output) for the duration of the experiment; PVR was greater than $200 \mathrm{~mm} \mathrm{Hg/L/min.} \mathrm{At} 30$ minutes after revascularization, when the recipient's native lung was clamped, blood flow to the transplanted lung remained minimal, and PVR exceeded $400 \mathrm{~mm} \mathrm{Hg} / \mathrm{L} / \mathrm{min}$, which was associated with immediate hemodynamic collapse. Arterial oxygen tension measured from arterial blood ranged between 60 and $90 \mathrm{~mm} \mathrm{Hg}$ on $100 \%$ oxygen.

The right pulmonary artery was reclamped periodically over the next 90 minutes, but flow to the transplanted lung remained low, and the animal was put to death.

The hMCP lung recipient treated with bivalirudin exhibited blood flow to the transplanted left lung $(0.45 \mathrm{~L} / \mathrm{min})$ that was $40 \%$ of cardiac output $(1.1 \mathrm{~L} / \mathrm{min})$. When the right pulmonary artery was snared, the left lung accommodated 


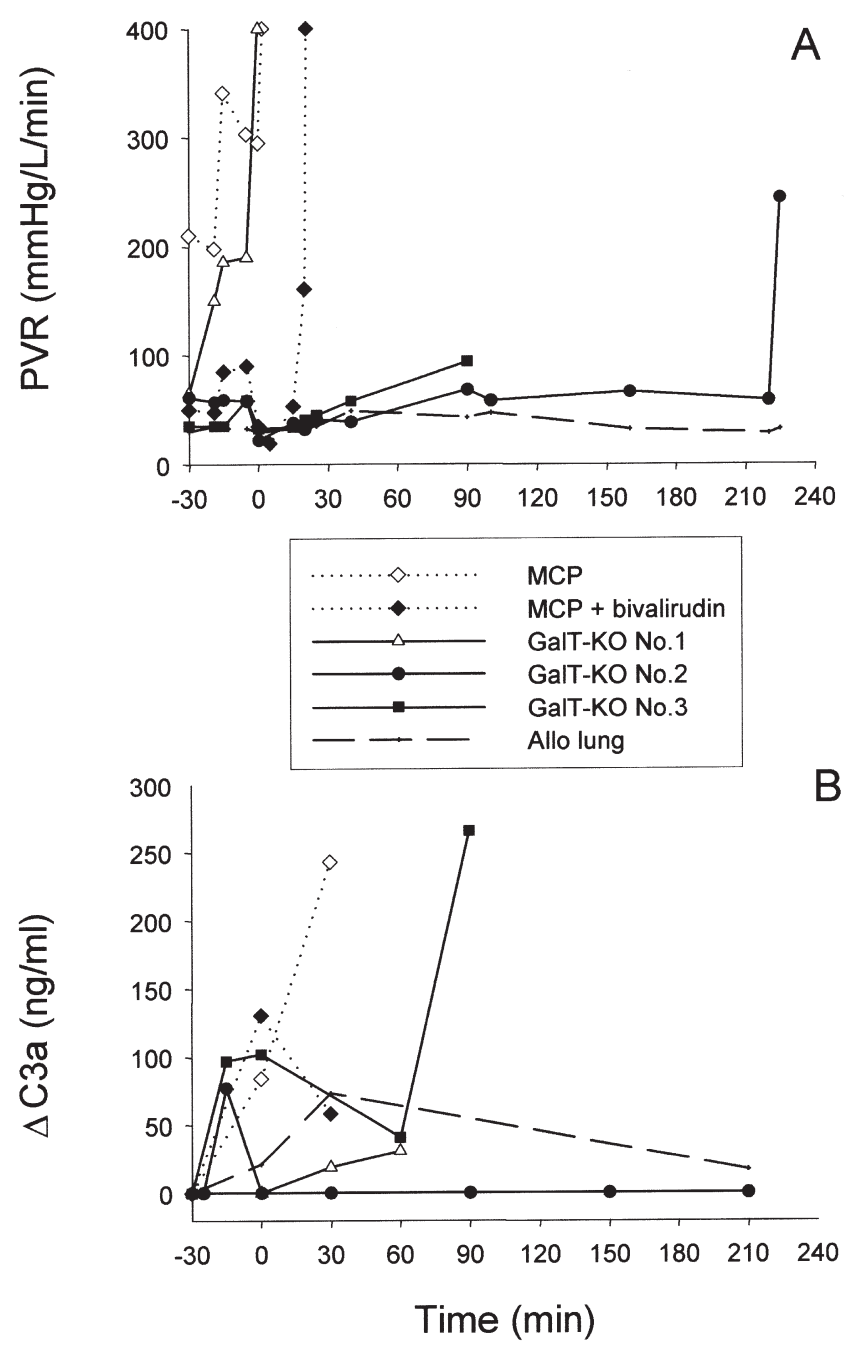

Figure 1. Pulmonary vascular resistance (PVR) (A) and plasma levels of complement fragments C3a (B) of all lung recipients. Time $-\mathbf{3 0}$ represents the initial revascularization of the grafts. Time $\mathbf{0}$ marks the time when right pulmonary artery to the native lung was clamped and the life-supporting function of the grafts was assessed. C3a levels are expressed as the amount of complement fragments produced above the baseline $(\Delta)$. The legend in this figure applies to all following figures. The long-dash line represents the allogenic lung. Two short-dash lines stand for membrane cofactor protein (MCP) lungs, and three solid lines for galactosyl transferase knockout (GaIT-KO) lungs. Open symbols represent nonfunctional lungs and filled symbols represent lungs that were able to support life.

the full cardiac output of $1.0 \mathrm{~L} / \mathrm{min}$, and the transplanted lung supported the recipient life for a total of 21 minutes. Ultimately, hypoxemia (arterial oxygen tension $35 \mathrm{~mm} \mathrm{Hg}$ ) was associated with bradycardia and hypotension and led to the termination of the experiment.
TABLE 1. Summary of experimental outcomes

\begin{tabular}{llrc}
\hline & & \multicolumn{2}{c}{ Graft survival } \\
\cline { 3 - 4 } $\begin{array}{l}\text { Treatment } \\
\text { group }\end{array}$ & Cause of failure & $\begin{array}{c}\text { Total } \\
\text { (min) }\end{array}$ & $\begin{array}{c}\text { Life-supporting } \\
\text { (min) }\end{array}$ \\
\hline MCP & Poor flow & $<30$ & 0 \\
MCP + & High PVR & 51 & 21 \\
$\quad$ bivalirudin & & & \\
GalT-KO No. 1 & Poor flow & $<30$ & 0 \\
GalT-KO No. 2 & Tracheal edema & 255 & 225 \\
GalT-KO No. 3 & Tracheal edema & 120 & 90 \\
Allogenic & Elective termination & $24 \mathrm{~h}$ & $24 \mathrm{~h}$
\end{tabular}

MCP, Membrane cofactor protein; GalT-KO, galactosyl transferase knockout; $P V R$, peripheral vascular resistance.

One GalT-KO lung (No. 1) failed to support life owing to high PVR. The other two GalT-KO lungs (Nos. 2 and 3) were life-supporting for 225 and 90 minutes, respectively. The recipient of GalT-KO No. 2 lung exhibited tracheal edema at 2 hours, associated with decline in arterial oxygen tension from 390 to $84 \mathrm{~mm} \mathrm{Hg}$; arterial oxygen tension remained low for the remainder of the experiment. PVR remained low and cardiac output stable for approximately 4 hours, when pulmonary flow dropped from 0.5 to $0.2 \mathrm{~L} / \mathrm{min}$. During the last 5 minutes of the experiment, systemic blood pressure dropped and the animal died. GalT-KO No. 3 lung survived 90 minutes with physiology similar to the lung allograft (PVR remained low at $100 \mathrm{~mm} \mathrm{Hg} / \mathrm{L} / \mathrm{min}$ ), but progressive tracheal edema impeding ventilation and oxygenation led to recipient death. Of note, this recipient had some native lung pulmonary edema before xenograft revascularization. The cause of the acute injury to the native lung in this instance is unknown.

\section{Complement Activation}

Relative to prolific complement activation seen during perfusion of wild-type lungs ex vivo $(>1000 \mathrm{ng} / \mathrm{mL}),{ }^{4}$ in vivo perfusion of five genetically modified lungs in this experience was associated with minimal increase in $\mathrm{C} 3 \mathrm{a}$ (Figure 1, B). Lung C4d and C5b-9 deposition was minimal at all time points (not shown), suggesting that in vivo perfusion of these lungs with baboon blood did not result in significant complement activation systemically or in the swine lung.

\section{Platelet Sequestration and Activation Markers}

Platelet counts were minimally perturbed after graft revascularization and institution of life-supporting lung function by the allograft (Figure 2, A), and there was no detectable platelet activation (CD62P expression in circulating platelets, Figure 2, $B ; \beta$ TG elaboration, Figure 2, C). In contrast, platelet counts fell by $18 \%$ to $60 \%$ after xenograft reperfusion and/or conversion to life-supporting graft perfusion, accompanied by varying 

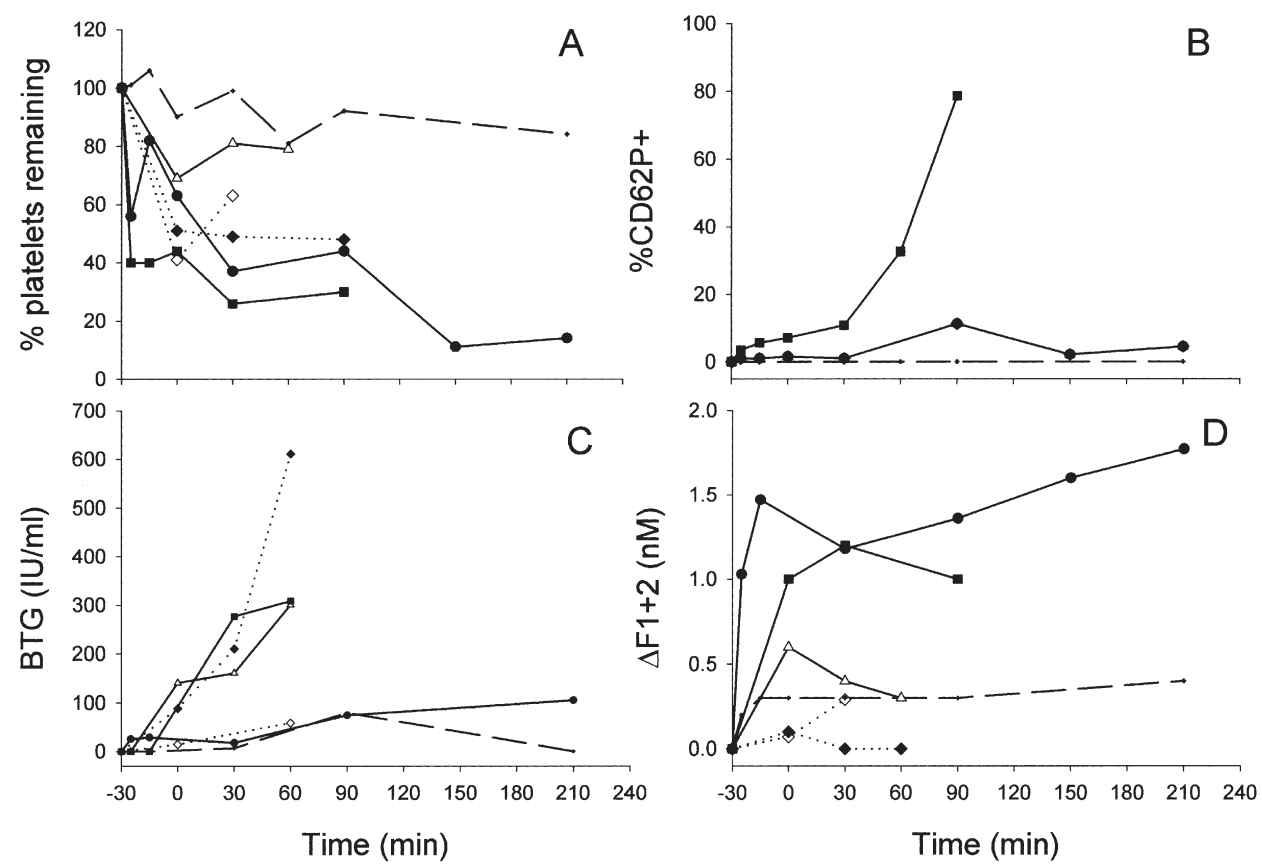

Figure 2. Platelet and coagulation cascade activation profile of all lung recipients. Symbols correlate with those described in Figure 1. Platelet activation was assessed by quantifying platelet sequestration from circulation measured by automated complete blood count (A), expression of CD62P marker (B), and release of betathromboglobulin (BTG) from $\alpha$ granules (C). Activation of the coagulation cascade was detected by the formation of thrombin measured by fragments $1+2(F 1+2)(D)$. All data are expressed as change from the baseline $(\Delta)$.

degrees of activation in residual circulating platelets. $\mathrm{hMCP}$ expression or additional thrombin inhibition did not prevent platelet sequestration or activation. GalT-KO No. 1, which had minimal graft perfusion, exhibited little decline in platelet number after reperfusion and partial recovery during the observation interval. GalT-KO lungs 2 and 3 exhibited a sharp initial fall in circulating platelet number after xenograft reperfusion and a further subsequent decline over 90 to 225 minutes of life-supporting lung xenograft function. Activation of those platelets remaining in the circulation was minimal in the recipient of GalT-KO lung No. 2, and marked (78\% at 90 minutes) in the recipient of GalT-KO No. 3. In the three experiments in which both CD62P expression and $\beta$ TG were assayed, results were concordant.

\section{Coagulation Activation Marker-F1+2 and Thrombin Formation}

Significant thrombin formation $(\mathrm{F} 1+2$ increase $>0.2$, Figure $2, D$ ) was observed after lung allotransplantation and in every lung xenograft except for the hMCP lung treated with the direct thrombin inhibitor bivalirudin. That experiment aside, higher thrombin levels were measured in association with physiologic xenograft perfusion. Interestingly, although the allogenic lung showed a slight rise in thrombin formation after reperfusion, no further increase was detected throughout the next 24 hours (data after 4 hours are not shown).

\section{Blood Cell Counts}

Neutrophil counts fell with high PVR and low transpulmonary blood flow through the MCP lung without bivalirudin treatment, but not in the allograft that had physiologic transpulmonary blood flow (Figure 3). Neutrophil counts declined $35 \%$ to $45 \%$ after reperfusion of GalT-KO lungs 2 and 3, which subsequently supported life, but not in the life-supporting bivalirudin-treated MCP lung or in GalT-KO lung 1, which did not support life. No consistent trend was manifest in association with institution of life-supporting function or subsequent graft failure. Circulating monocyte counts fell $30 \%$ to $70 \%$ during initial reperfusion of all grafts with physiologic blood flow (bivalirudin-treated MCP, GalT-KO Nos. 2 and 3, as well as the allograft) and tended to fall in association with xenograft failure except in the instance in which thrombin was inhibited.

\section{Histology and Immunohistochemistry}

Histologic examination of lung biopsy specimens by hematoxylin and eosin staining is shown in Figure 4. The allogenic lung at 24 hours retained normal microscopic anatomy with clear 

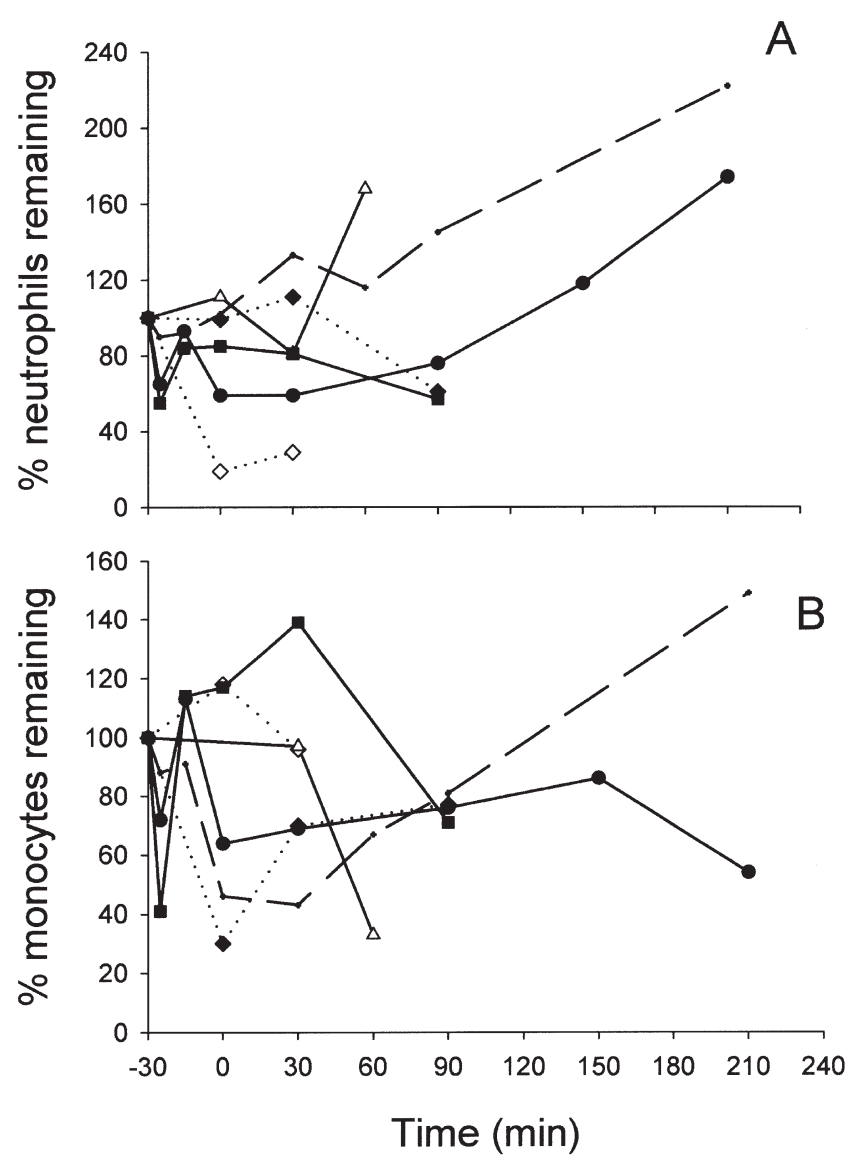

Figure 3. Neutrophil (A) and monocyte (B) sequestration from circulation. Symbols correlate with those described in Figure 1. Cell numbers are expressed as percent remaining in circulation using the cell counts before graft revascularization as the baseline.

alveoli, thin septae, and no intravascular thrombosis (Figure 4, A). There was severe intra-alveolar, interstitial, and intratracheal hemorrhage in hMCP lung treated with bivalirudin, a feature unique to this recipient. At failure, GalT-KO lungs exhibited prevalent intravascular thrombosis (Figure 4, $C$ ) and prominent septal vascular congestion (Figure $4, D$ ). Immunohistochemical staining of lung biopsy specimens for platelet deposition (CD41) is shown in Figure 5. Allogenic lung at 24 hours showed no platelet deposition, as expected (Figure 5, A and $B$ ). Likewise, GalT-KO lung at 30 minutes showed minimal platelet deposition (Figure 5, $C$ and $D$ ). In contrast, GalT-KO lung at failure showed intense platelet deposition associated with von Willebrand factor positive endothelial structures (Figure 5, $E$ and $F$ ).

\section{Discussion}

This in vivo lung xenograft experience using three GalT-KO and two hMCP grafts confirms and extends our mechanistic understanding of acute lung xenograft injury based on an ex vivo perfusion model with human blood. Two of three GalT-KO lungs supported the recipient in a stringent, life-supporting single-lung transplant model. Failure of these grafts was associated with direct (thrombin formation, intravascular platelet deposition) and indirect (platelet activation) evidence of intravascular thrombosis, just as in our previous report. ${ }^{19}$ Similarly, we find that direct thrombin inhibition significantly improves transpulmonary blood flow and permits transient life-supporting function of a baboon by a swine lung expressing a human complement regulatory protein, hMCP. However, by itself, hMCP expression was not sufficient to support life in one experiment.

As part of our ongoing studies using a different model, the right lungs of the GalT-KO No. 2 and No. 3 experiments were perfused ex vivo with human blood (manuscript in preparation). Interestingly, in these ex vivo experiments, both the tempo of graft failure ( $>6$ hours in GalT-KO No. 2 and 56 minutes for GalT-KO No. 3) and mode of graft failure (sudden loss of transpulmonary graft flow versus loss of pulmonary capillary barrier function, respectively) were similar for each pair of lungs studied contemporaneously in both in vivo and ex vivo experiments. The histologic features of ex vivo perfused lungs also showed intravascular thrombosis at the time of graft failure. These preliminary observations suggest that our ex vivo model is useful for predicting in vivo lung xenograft behavior, while illustrating the importance of confirming hypotheses developed with ex vivo perfusion in a life-supporting model.

In outbred swine, Yeatman, ${ }^{9,10}$ Kulick, ${ }^{11}$ Gonzalez-Stawinski, ${ }^{20}$ and their colleagues have previously obtained prolonged lung function by inhibiting complement, adsorbing anti-swine antibody, and supporting animals with high-dose pressors. However, these models only rendered the recipient intermittently dependent on function of the graft. $^{9-11,20}$ Although constructs such as this can provide useful mechanistic information, in vivo models that do not mandate continuous life support and ex vivo perfusion systems using nonphysiologic perfusion criteria (low flow, high pressure, or perfusates lacking critical formed blood elements such as platelets or nucleated cells) may not accurately predict functional capacity of the graft in vivo. ${ }^{21}$

On the basis of $\mathrm{C} 3 \mathrm{a}$ elaboration and immunohistologic analysis, both hMCP and GalT-KO lung xenografts are well protected with respect to antibody-driven classic pathway complement activation. Although these genetic modifications are effective in blocking their targeted pathway, hyperacute rejection still occurs, presumably driven primarily by other, complement-independent pathways. ${ }^{22-24}$ Our current and prior observations suggest that efficient regulation of complement alone is inadequate to permit clinically useful function of porcine lung in a human blood environment. ${ }^{4,22-24}$ 

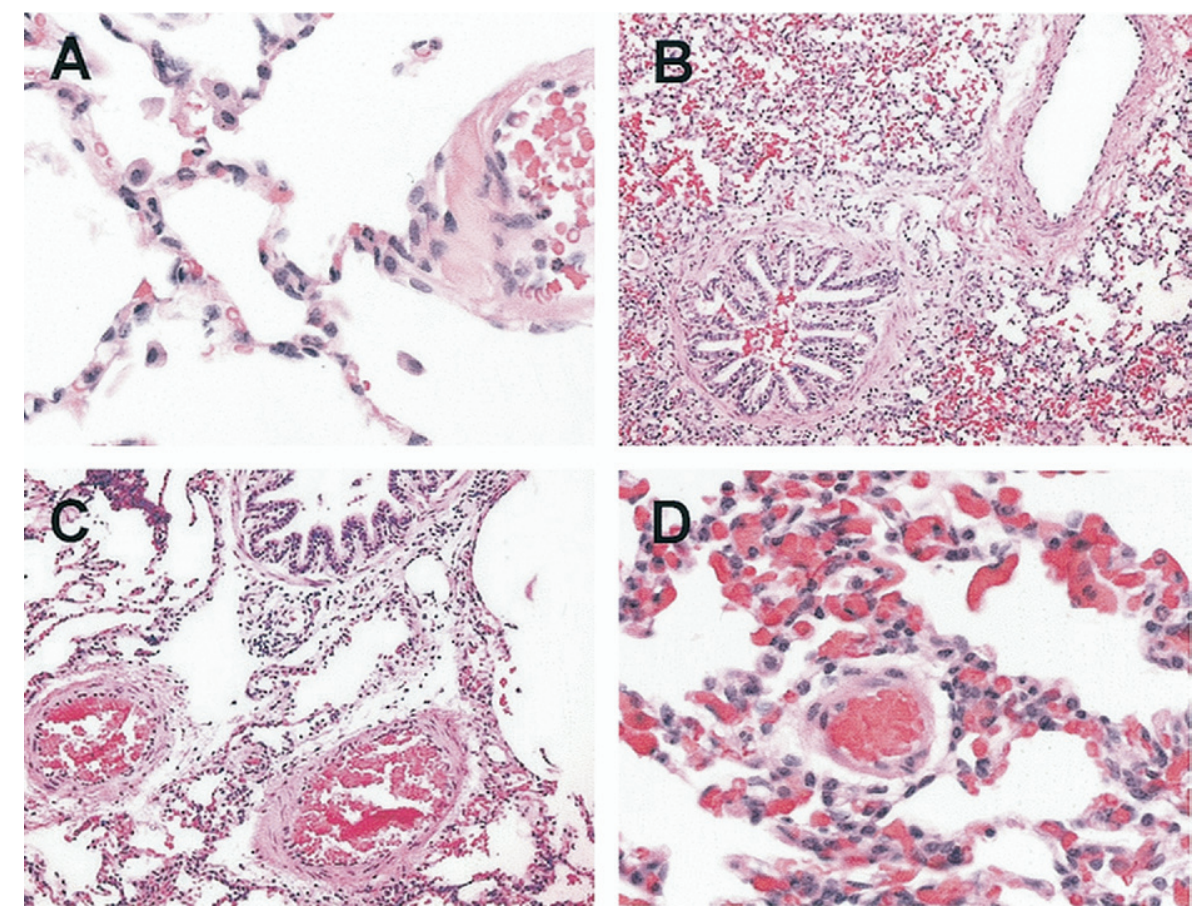

Figure 4. Hematoxylin and eosin histologic studies of lung biopsy specimens. Allogenic lung (A) at 24 hours showed normal lung histologic features with thin alveolar septae and no pulmonary edema or intravascular thrombosis. Membrane cofactor protein lung + bivalirudin (B) showed severe interstitial/alveolar hemorrhage extending to the airway but no intravascular thrombosis. GalT-KO lung showed significant intravascular thrombosis (C) and severe septal vascular congestion (D) at failure.

Platelet and coagulation activation occur consistently in the context of discordant organ xenotransplantation. ${ }^{25-28}$ Whereas the lung allograft recipient had minimal platelet sequestration and only a modest degree of thrombin formation, in all three recipients of physiologically reperfused lung xenografts $30 \%$ to $60 \%$ of platelets were sequestered from the circulation within 30 minutes after revascularization, and thrombin formation was also high in all three GalT-KO lung recipients. Intravascular thrombosis and tissue platelet deposition at the time of lung failure further strongly support our working hypothesis that coagulation activation is the cause of ultimate graft demise. However, since we have not examined potential differences in levels of donor-reactive anti-swine antibodies among the primate recipients, it remains possible that anti-swine antibodies (anti-Gal for MCP lungs and anti-non Gal for GalT-KO lungs) might serve as the primary initiator of the events that ultimately lead to lung injury. Nonetheless, it appears that, to achieve long survival of GalT-KO lungs, the etiology of coagulation dysregulation needs to be addressed independently from complement activation.

Coagulation cascade activation can be prevented either directly or indirectly at the level of thrombin formation and/or platelet activation. In this study, bivalirudin, a direct thrombin inhibitor, controlled thrombin production, limiting the rise in PVR, and prolonged life-supporting time. This replicates our previous experience ex vivo where bivalirudin significantly improved mean lung survival, from 8 to 77 minutes, even in the context of intact antibody and complement-driven mechanisms. ${ }^{29}$ Importantly, bivalirudin also consistently delayed complement activation, similar to combined platelet receptor blockade. $^{22}$ We infer that endothelial thrombin formation, which occurs in the context of xenotransplantation even with quiescent endothelial cells, ${ }^{27}$ may be a crucial primary stimulus for the initiation of platelet aggregation and activation, and thus for complement cascade amplification during hyperacute lung rejection, and may be expected to occur even if antibody and complement are removed from the equation. Although thrombin is one important platelet activator, there are many other agonists/platelet receptor pairs that likely will need to be considered, including von Willebrand factor and glycoprotein Ib.

Although bivalirudin effectively blocked thrombin formation and intravascular thrombosis in the hMCP graft, lung failure was associated with interstitial and alveolar hemorrhage, indicative of profound loss of endothelial barrier function. If caused by antibody-mediated injury, as seems probable, GalT-KO lungs should be less susceptible to this phenomenon.

Peterson and associates ${ }^{30}$ demonstrated that human monocytes adhere to swine endothelial cells by binding to the $\alpha$-Gal antigen, which not only causes monocyte sequestration in the tissue but also acts as a trigger to upregulation of endothelial cells' adhesion molecule, cytokine, and tissue factor expression. It is interesting that, in this study, we found sequestration of monocytes from circulation in all four functional lungs, 

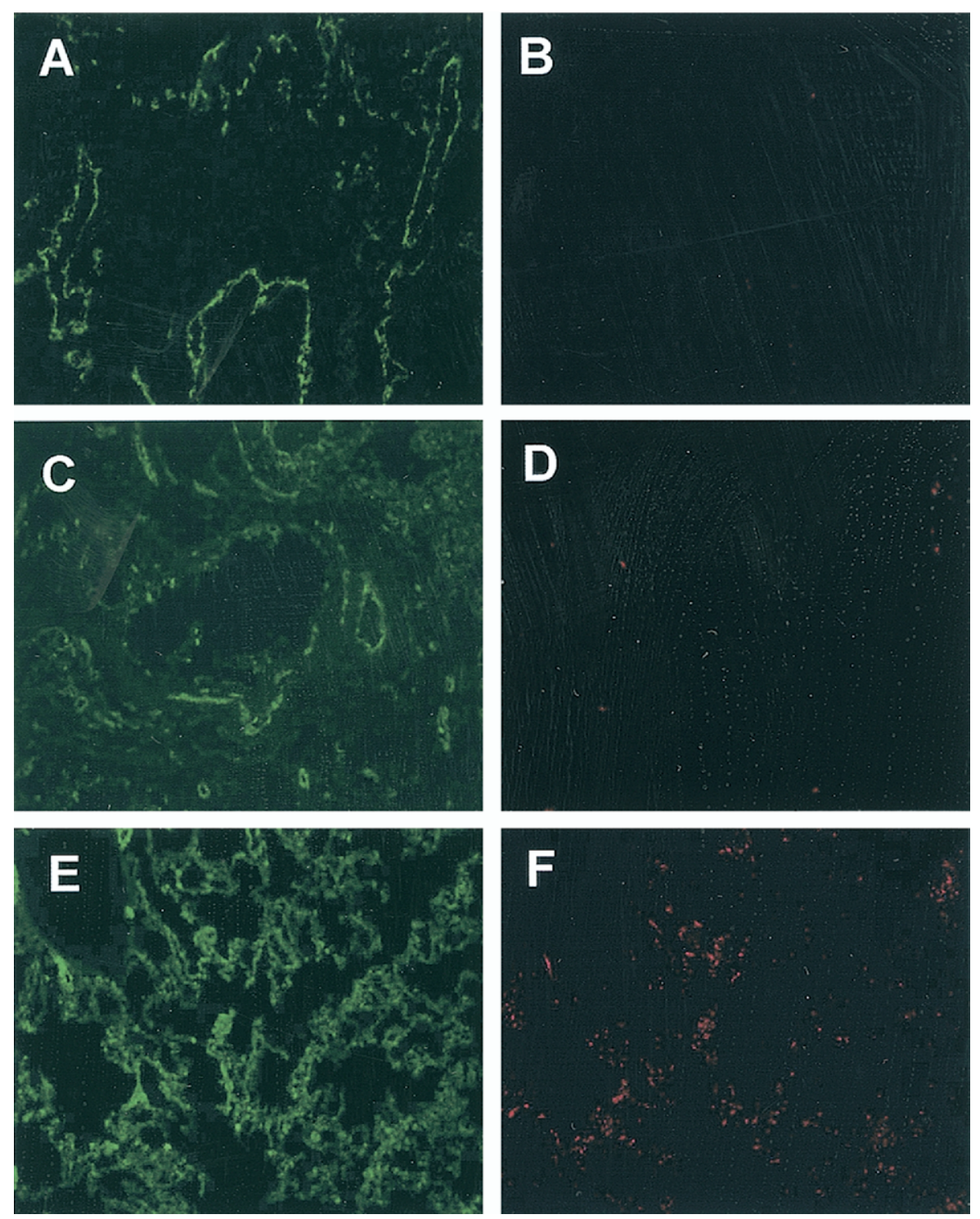

Figure 5. Immunohistochemistry staining for platelet deposition (CD41) of lung biopsy specimens. The green and red colors represent von Willebrand factor (endothelial marker) and CD41 (platelet marker), respectively. Allogenic lung (A and B) at 24 hours showed essentially no platelet deposition. Likewise, GaIT-KO lungs at $\mathbf{3 0}$ minutes (C and D) exhibited minimal platelet deposition. However, at lung failure ( $E$ and $F$ ), there was prominent platelet deposition (red) in association with alveolar septal endothelium (green) in the GalT-KO lung. including the allogeneic lung and the two GalT-KO lungs. However, more data are needed to confirm these observations and discern their physiologic significance.

In summary, we have shown that whereas human complement regulatory protein transgenic lungs failed to support life without additional treatment to the recipient, GalT-KO lungs were able to do so for as long as 3.5 hours. Our associated findings support our working paradigm that dysregulated coagulation participates directly in GalT-KO lung injury. Our model postulates that primate monocytes and porcine intravascular macrophages trigger local coagulation pathway activation through release of tissue factor when activated by antibody, complement, or local thrombin formation. Tissue factor in turn initiates and amplifies platelet aggregation as well as activation of endothelial cells and a plethora of other cell types in lung and blood. Dysfunctional thromboregulatory processes in the setting of xenotransplantation fail to abort amplification of several prothrombotic cascades, ${ }^{25-28}$ leading to prolific intravascular coagulation and concomitant complement activation. In our estimation, improved mechanistic definition of the interaction between these various triggers - antibody, complement, adhesion molecules, platelets, and other coagulation pathway constituents-will be necessary to devise efficient, practical strategies for aborting the various pathogenic positive feedback loops outlined here, and thus to develop clinically suitable approaches for lung xenografting.

The work is supported by kind gifts from INO Therapeutics for nitric oxide gas supply and accessories, Boehringer Ingleheim for 
C1-inhibitor, the Medicines Company for bivalirudin, and the University of Maryland's General Clinical Research Centers for nursing staff and financial support in collecting blood from human donors for parallel ex vivo lung perfusion experiments. We thank the Mayo Clinic for their support with MCP transgenic swine and Immerge Biotherapeutics and Revivicor for supplying GalT-KO lungs. We thank Stuart L. Houser, MD, for his independent review of the immunohistologic primary data and associated interpretations and Amal Laaris, Qi Feng, Chris Avon, and Nitin Sangrampurkar for their valuable technical assistance.

\section{References}

1. Leventhal JR, John R, Fryer JP, Dalmasso A, Matas AJ, Bolman RM, et al. Removal of baboon and human antiporcine $\operatorname{IgG}$ and $\operatorname{IgM}$ natural antibodies by immunoadsorption. Transplantation. 1995;59:294-300.

2. Azimzadeh A, Meyer C, Watier H, Jaeck D, Cinqualbre J, Wolf P, et al. Removal of primate xenoreactive natural antibodies by extracorporeal perfusion of pig kidneys and livers. Transplant Immunol. 1998; 6:13-22.

3. Katopodis AG, Warner RG, Duthaler RO, Davies HF, Manez R, White $\mathrm{D}$, et al. Removal of anti-Galalpha1,3Gal xenoantibodies with an injectable polymer. J Clin Invest. 2002;110:1869-77.

4. Azimzadeh A, Zorn GL, Blair KS, Cozzi E, White DJ, Pierson RN 3rd, et al. Hyperacute lung rejection in the pig-to-human model. 2. Synergy between soluble and membrane complement inhibition. Xenotransplantation. 2003;10:120-31.

5. Schroeder C, Pfeiffer S, Wu G, Zorn GL 3rd, Ding L, Allen C, et al. Effect of complement fragment 1 esterase inhibition on survival of human decay-accelerating factor pig lungs perfused with human blood. J Heart Lung Transplant. 2003;22:1365-75.

6. Cozzi E, White DJG. The generation of transgenic pigs as potential organ donors for humans. Nat Med. 1995;1:964-6.

7. Zaidi A, Schmoeckel M, Bhatti F, Wallwork J, White D, Friend P, et al. Life-supporting pig-to-primate renal xenotransplantation using genetically modified donors. Transplantation. 1998;65:1584-90.

8. Daggett CW, Yeatman M, Lodge AJ, Frank MM, Platt JL, Davis RD, et al. Total respiratory support from swine lungs in primate recipients. J Thorac Cardiovasc Surg. 1998;115:19-27.

9. Yeatman M, Daggett CW, Lau CL, Logan JS, Platt JL, Davis RD, et al. Human complement regulatory proteins protect swine lungs from xenogeneic injury. Ann Thorac Surg. 1999;67:769-75.

10. Yeatman M, Daggett CW, Parker W, Logan JS, Platt JL, Davis RD, et al. Complement-mediated pulmonary xenograft injury: studies in swine-to-primate orthotopic single lung transplant models. Transplantation. 1998;65:1084-93.

11. Kulick DM, Salerno CT, Dalmasso AP, Paz MG, Fodor WL, Bolman RM 3rd. Transgenic swine lungs expressing human CD59 are protected from injury in a pig-to-human model of xenotransplantation. J Thorac Cardiovasc Surg. 2000;119:690-9.

12. Lau CL, Daggett WC, Yeatman MF, Parker W, Platt JL, Davis RD, et al. The role of antibodies in dysfunction of pig-to-baboon pulmonary transplants. J Thorac Cardiovasc Surg. 2000;120:29-38.

13. Blum MG, Chang AC, Collins BH, Knaus SA, Christman BW, Pierson RN 3rd. The effect of nitric oxide and thromboxane blockade on pulmonary vascular resistance in a pig to primate lung transplant model. The Fourth International Congress for Xenotransplantation; 1997; Nantes, France: abstract, p. 290.

14. Azimzadeh A, Schroeder C, Pierson RN 3rd. Lung xenotransplantation: In: Banner N, editor. Lung transplantation. 1st ed. Cambridge [UK]: Cambridge University Press; 2003. p. 374-85.

15. Kolber-Simonds D, Lai L, Watt SR, Forsberg EJ, Prather RS, Hawley RJ, et al. Production of alpha-1,3-galactosyltransferase null pigs by means of nuclear transfer with fibroblasts bearing loss of heterozygosity mutations. Proc Natl Acad Sci U S A. 2004;101:7335-40.

16. Ayares D, Colman A, Dai Y, Shiels P, Moore M. Cloning pigs deficient in alpha 1,3 galactosyltransferase. Graft. 2001;4:80-2.

17. Tseng YL, Kuwaki K, Dor FJ, Schuurman HJ, Sachs DH, Cooper DK, et al. Alpha 1,3-galactosyltransferase gene-knockout pig heart trans- plantation in baboons with survival approaching 6 months Transplantation. 2005;80:1493-500.

18. Yamada K, Yazawa K, Shimizu A, Awwad M, Sykes M, Sachs DH, et al. Marked prolongation of porcine renal xenograft survival in baboons through the use of alpha 1,3-galactosyltransferase geneknockout donors and the cotransplantation of vascularized thymic tissue. Nat Med. 2005;11:32-4.

19. Schroeder C, Allan JS, Nguyen BN, Shuurman H-J, Sachs DH, Pierson RN 3rd. Hyperacute rejection is attenuated in GalT-KO swine lungs perfused ex vivo with human blood. Transplant Proc. 2005;37:512-3.

20. Gonzalez-Stawinski GV, Daggett CW, Lau CL, Gaca JG, Parker W, Davis RD Jr, et al. Non-anti-Gal alpha1-3Gal antibody mechanisms are sufficient to cause hyperacute lung dysfunction in pulmonary xenotransplantation. J Am Coll Surg. 2002;194:765-73.

21. Kaplon RJ, Platt JL, Kwiatkowski PA, Shah AS, Masroor S, Michler RE. Absence of hyperacute rejection in pig-to-primate orthotopic pulmonary xenografts. Transplantation. 1995;59:410-6.

22. Pfeiffer S, Zorn GL 3rd, Zhang JP, Robson SC, Azimzadeh AM, Pierson RN 3rd. Hyperacute lung rejection in the pig-to-human model. III. Platelet receptor inhibitors synergistically modulate complement activation and lung injury. Transplantation. 2003;75:953-9.

23. Nguyen BN, Zwets E, Schroeder C, Pierson RN 3rd, Azimzadeh AM Beyond antibody-mediated rejection: hyperacute lung rejection as a paradigm for dysregulated inflammation. Curr Drug Targets Cardiovasc Haematol Disord. 2005;5:255-69.

24. Collins BJ, Blum MG, Parker RE, Zorn GL, Chrisman BW, Pierson $\mathrm{RN}$. Thromboxane mediates pulmonary hypertension and contributes to lung inflammation during hyperacute lung rejection. J Appl Physiol. 2000;90:2257-68.

25. Siegel JB, Grey ST, Lesnikoshi B-A, Kopp CW, Soares M, Robson SC. Xenogeneic endothelial cells activate human prothrombin. Transplantation. 1997;64:888-96.

26. Robson SC, Cooper DK, d'Apice AD. Disordered regulation of coagulation and platelet activation in xenotransplantation. Xenotransplantation. 2000;7:166-76.

27. Jurd KM, Gibbs RV, Hunt G. Activation of human prothrombin by porcine aortic endothelial cells - a potential barrier to pig to human xenotransplantation. Blood Coag Fibrinol. 1996;7:336-43.

28. Chen D, Riesbeck K, McVey JH, Tuddenham EG, Lehler RI, Dorling A, et al. Human thrombin and FXa mediate porcine endothelial cell activation, modulation by expression of TFPI-CD 4 and hirudin-CD4 fusion proteins. Xenotransplantation. 2001;8:258-65.

29. Zorn GL, Pfeiffer S, Azimzadeh AM, Pierson RN 3rd. Thrombin inhibition protects the pulmonary xenograft from hyperacute rejection. Surg Forum. 2000;LI:338-40.

30. Peterson MD, Jin R, Hyduk S, Duchesneau P, Cybulsky MI, Waddell TK. Monocyte adhesion to xenogeneic endothelium during laminar flow is dependent on alpha-gal-mediated monocyte activation. $J$ Immunol. 2005;174:8072-81.

\section{Discussion}

Dr Joseph B. Zwischenberger (Galveston, Tex). As one approaches the problem of an off-the-shelf artificial lung, the spectrum ranges from aplastic artificial lung all the way to transspecies transplant. You are to be congratulated for persisting in your work of trying to accomplish transspecies transplantation, which has the promise for an off-the-shelf artificial lung.

This work has been ongoing for 10 or 15 years, and it strikes me that creating a transgenic knockout swine model is very difficult. You did not mention what it takes to create that. Could you please recap that for us?

Second, I am having some difficulty in trying to put together the accomplishment of a 2-hour survival in two of three pigs. How is two of three an advance?

Finally, you mentioned intravascular coagulation as being the hallmark of the failure of these transplanted lungs, and you mentioned platelet activation and intravascular thrombosis. Could you 
tease out which is the chicken and which is the egg? Are you activating platelets, which are then causing intravascular coagulation? Are you causing intravascular coagulation, which is then activating platelets? It may help to define your future strategies.

Dr Nguyen. Thank you, Dr Zwischenberger. To answer your first question about how to make the GalT-KO lungs, I wish I knew because then I would be able to make them myself and make them more available. These are very precious animals because they are genetically engineered by only a few groups and we have to share them among many investigators in the field. My understanding is that they can either be cloned or bred naturally. The problem is that they do not breed as well as the other domestic swine and sometimes they have a higher rate of perinatal mortality as well. However, if they survive to adulthood, then they have no other health problems so far as we know.

To answer your second question about whether two of three GalT-KO lungs surviving at 4 hours is an achievement, I think this is phenomenal. If you attach wild-type lungs or even transgenic MCP lungs untreated to a baboon, you do not have any lifesupporting function beyond minutes. In the past many other investigators did in vivo transplant. You have to do many treatments to the recipient in terms of antibody absorption before surgery. A lot of manipulation to the recipient sometimes is not beneficial to the recipient. Thus I think two of three lungs beyond 1 hour and 4 hours is significant.
Your third question concerned intravascular thrombosis and whether the coagulation activation starts first or platelet activation starts first. I think it is more of a circle feedback loop. If you perfuse human blood to a pig organ, even in the nonactivated pig endothelial cells, you do see human prothrombin converted to thrombin, because pig thrombomodulin is so ineffective at preventing this process. Human thrombomodulin is very effective in doing this, but not pig thrombomodulin. Thus you have thrombin formation. Even in the absence of complement activation, you have thrombin formation first, and then you could trigger coagulation activation directly from thrombin. However, then thrombin can also activate platelets, and activated platelets can trigger coagulation activation as well. Again, I think it is more like a circle feedback loop. We have to address both platelet activation and coagulation activation together to prevent intravascular thrombosis.

Dr Young Tae Kim (Seoul, South Korea). Did you look at the von Willebrand factor? We have been doing similar experiments, and what we found is that when we deplete the von Willebrand factor by giving vasopressin, the success rate increases. Did you check it?

Dr Nguyen. Do you mean check for the absence of GalT antigen in GalT-KO pigs?

Dr Kim. The von Willebrand factor.

Dr Nguyen. Yes. We did not do this ourselves, but the supplier of the GalT-KO pigs actually confirmed that. Every time they sent us the pigs, they confirmed that this is indeed GalT-KO. 